\title{
Increased circulating chemerin in patients with advanced carotid stenosis
}

\author{
Adrian Kammerer ${ }^{1 \dagger}$, Holger Staab ${ }^{2 \dagger}$, Maria Herberg ${ }^{3}$, Christine Kerner $^{1}$, Nora Klöting ${ }^{4}$ and Gabriela Aust ${ }^{*}$ (D)
}

\begin{abstract}
Background: Chemerin is an adipokine which plays a crucial role in atherosclerosis. Here, we examined whether circulating chemerin is enhanced in patients with advanced carotid stenosis.

Methods: Chemerin was quantified in 178 patients prior to carotid end arterectomy (CEA) and in age- and gendermatched controls $(n=163)$. Chemerin levels were related to anthropometric, clinical and metabolic characteristics of the patients.

Results: Chemerin levels were higher in patients compared to controls $(p<0.001)$. Chemerin correlated to parameters associated with inflammation such as $C$-reactive protein (CRP, $p<0.001)$, leukocyte blood count $(p<0.001)$ and circulating TNF-a $(p=0.004)$ in the patients. Chemerin levels did not differ between asymptomatic $(n=93)$ and symptomatic patients who experienced an ischemic event within 6 months prior to CEA $(n=85)$. However, in the case of high-grade carotid stenosis ( $\geq 90 \%)$, chemerin levels were higher in symptomatic $(n=44)$ compared to asymptomatic patients $(n=41, p=0.014)$. Chemerin was increased in patients with $(n=50)$ compared to patients without $(n=128)$ coronary artery disease $(C A D, p=0.002)$. A high level of chemerin increases the risk for CAD in patients $(p=0.0013)$.
\end{abstract}

Conclusions: Circulating chemerin is increased and correlates to inflammatory parameters in patients with advanced carotid stenosis.

Keywords: Chemerin, Carotid stenosis, Coronary artery disease

\section{Background}

Cardio- and cerebrovascular diseases are the primary cause for mortality worldwide with 17.3 million deaths per year [1]. Stroke is the leading cause of acquired disability in adults and the second leading cause of death in patients aged $>60$ years [2]. $87 \%$ of all strokes are ischemic due to the lack of blood flow, and $20 \%$ of all ischemic strokes result from a stenosis of extracranial vessels that nourish the brain such as the internal carotid artery $[3,4]$. In most cases, the underlying cause of cardio- and cerebrovascular events is atherosclerosis, a chronic inflammatory disease leading to arterial luminal narrowing. In Germany up to one million elderly people have a stenosis grade of the carotid artery $>50 \%$ [5]. Major risk

\footnotetext{
* Correspondence: gabriela.aust@medizin.uni-leipzig.de

${ }^{\dagger}$ Equal contributors

${ }^{1}$ Research Laboratories; Clinic for Visceral, Transplantation, Thoracic and Vascular Surgery, Leipzig University, Liebigstr. 19, D-04103 Leipzig, Germany Full list of author information is available at the end of the article
}

factors for atherosclerosis are adiposity, hypertension, diabetes, tobacco abuse and dysregulated blood lipids.

Adipose tissue secretes hormones and cytokines that regulate local and systemic metabolic processes and inflammatory responses. Chemerin is an adipokine first described to be located in inflammatory cells [6] and to chemoattract antigen-presenting cells as dendritic cells and macrophages [6]. Chemerin is the only known ligand for the $\mathrm{G}$ protein-coupled receptor chemokine-like receptor 1 (CMKLR1) and the $G$ protein-coupled receptor-1 (GRK1), and also binds to the chemokine receptor-like 2 (CCRL2). The majority of biological functions for chemerin have been attributed to activation of CMKLR1 [7]. Both, chemerin and CMKLR1 are present at high levels in adipose tissue regulating adipogenesis and adipocyte metabolism [8]. Moreover, CMKLR1 is expressed in the tunica media and endothelial layer of arteries, where chemerin contributes to vasoconstriction [9]. Chemerin is increased in the inflamed 
endothelial tissue where it exerts its chemotactic action and attracts further immune cells [10,11], and in foam and vascular smooth muscle cells of atherosclerotic lesions [3]. The data suggest a regulatory role of this adipokine in atherosclerosis.

Here, we examined whether chemerin contributes to atherosclerosis and the underlying inflammatory response. We quantified circulating chemerin in patients with advanced carotid stenosis prior to carotid endarterectomy (CEA) in age- and gender-matched controls and related chemerin levels to anthropometric, clinical and metabolic characteristics of the patients.

\section{Methods}

\section{Patients and controls}

In the first cohort, a total of 178 consecutive Caucasian men $(n=121)$ and women $(n=57)$ with extracranial carotid artery stenosis were included in the study. Patients, who were submitted for carotid endarterectomy (CEA) to the Clinic for Visceral, Transplant, Thoracic and Vascular Surgery (VTTG), Leipzig University, were recruited without any inclusion or exclusion criteria. According to the current valid guidelines of the European Society of Vascular Surgery (ESVS) [12] and the American Heart Association (AHA) [13], we graduated the luminal narrowing according to the NASCET criteria [14]. Two experienced vascular radiologists carried out assessments in consensus using axial source images as well as curved planar reformations and digital subtraction angiography.

Patients were classified into two groups, symptomatic $(n=85)$ or asymptomatic $(n=93)$, based on whether a patient had experienced an ischemic event (ipsilateral stroke, transient ischemic attack, or amaurosis fugax) within 6 months prior to CEA or not. Asymptomatic patients with carotid stenosis were identified randomly during clinical examination regarding coronary artery disease (CAD), peripheral artery disease, or an ischemic event in past medical history, that is $>6$ months from the date of admission to hospital. Furthermore, patients with carotid stenosis were classified into patients with CAD $(n=50)$ and no CAD $(n=128)$. Coronary angiography was performed in multiple projections. CAD was diagnosed as the presence of one or more vessels with $\geq 50 \%$ lumen narrowing.

In the second cohort, a total number of 163 control subjects were enrolled during adiposity consultation at the Department of Medicine, Leipzig University. Clinical parameters of this cohort were assessed as described previously [15].

\section{Blood samples and assays}

Peripheral venous blood was collected between 8 and 10 am after an overnight fast; in the case of carotid patients 1 day before CEA. Blood was immediately centrifuged at $2500 \mathrm{~g}$ for $20 \mathrm{~min}$. Plasma samples were stored at $-80{ }^{\circ} \mathrm{C}$ and thawed only once. Measurement of routine biochemical parameters was performed with standard procedures $[16,17]$. Chemerin was quantified with a sandwich ELISA (BioVendor GmbH, Kassel, Germany; sensitivity: $0.1 \mathrm{ng} / \mathrm{ml})$. TNF- $\alpha$ was measured using an ELISA (R\&D Systems, Inc., Minneapolis, USA; sensitivity: $0.1 \mathrm{ng} / \mathrm{ml}$, reference range of normal TNF- $\alpha-$ values: $<5 \mathrm{ng} / \mathrm{ml})$.

\section{Statistics}

Parameters were tested against a normal distribution using the one sample Kolmogorov-Smirnov test. Nonnormally distributed parameters were logarithmically transformed to approximate a normal distribution. Mean and standard error of mean (SEM), for non-normally distributed parameters median and the interquartile range [25th-75th percentiles], were used. A comparison between different groups regarding categorical variables was carried out using the Chi-square test. Two independent samples were compared using the Students's t-test for normally distributed data and the MannWhitney U-test for non-normally distributed data, respectively. Correlations between two quantitative variables were assessed using the Pearson coefficient for normally distributed data and Spearman Rho for nonnormally distributed data, respectively. Receiver Operating Characteristic (ROC) analysis was used to assess the diagnostic power of chemerin for discrimination between CAD and no CAD patients. The observed area under the ROC curve (AUC) was tested against the nullhypothesis $(\mathrm{AUC}=0.5)$ using the Mann-Whitney U-test. A logistic regression model was performed to further evaluate whether high chemerin increases the risk for CAD. All statistical computations were performed using SPSS version 24.0 (Chicago, IL, USA). $P$ values less than 0.05 were considered as significant.

\section{Results \\ Higher circulating chemerin levels in patients with advanced carotid stenosis}

Baseline information on anthropometric, clinical and metabolic characteristics of the 178 patients and 163 control subjects are shown in Table 1. Age and gender distribution did not differ between both groups, allowing for an unbiased comparison of the other parameters. The body mass index (BMI; $p=0.001)$, total cholesterol $(p=0.009)$, low density lipoprotein (LDL) cholesterol ( $\mathrm{p}$ $=0.001$ ), and high density lipoprotein (HDL) cholesterol $(p=0.005)$ were lower, and the leukocyte blood count ( $\mathrm{p}$ $=0.001), \operatorname{CRP}(p=0.010)$ and triglycerides $(p=0.042)$ were higher in patients compared to controls. $63.5 \%$ of the patients received statins, HMG-CoA reductase 


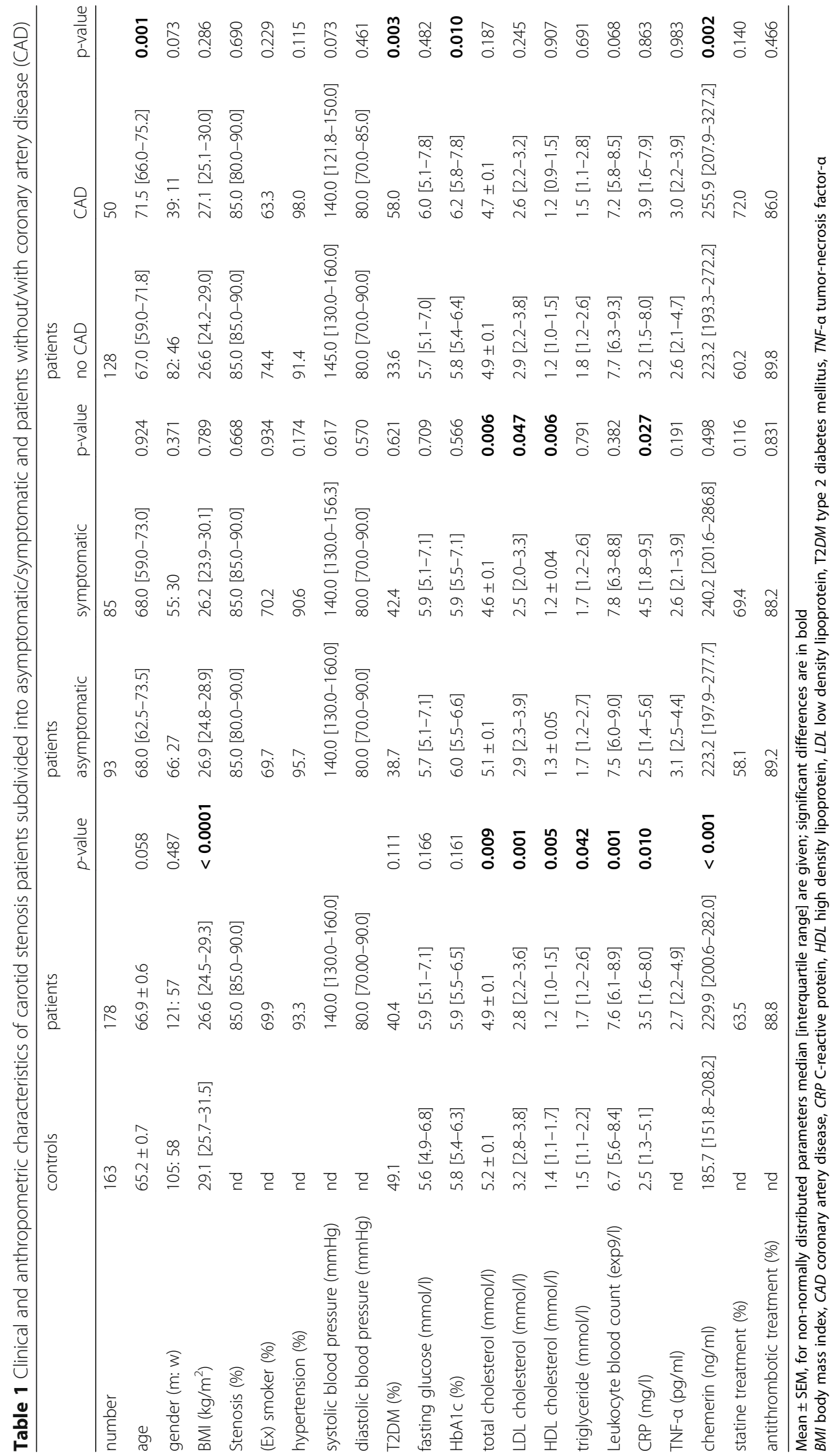


inhibitors, which effectively reduce circulating LDL cholesterol (statin-untreated 3.5 [3.0-4.1] $\mathrm{mmol} / \mathrm{l}$; statin treated 2.4 [2.1-3.1] mmol/l; $p<0.001)$. HbA1c $(p=0$. $161)$ and fasting glucose $(p=0.166)$ did not differ between both groups.

Circulating chemerin levels were increased 1.3 fold in patients compared to controls $(p<0.001$, Fig. 1$)$. Within the patient group women had higher chemerin levels (268.89 [215.1-319.92] ng/ml) compared to men (231.42 [193.00-267.82] ng/ml; $p<0.0001)$. Correlations of the chemerin level to patient characteristics are summarized in Table 2. Chemerin levels correlated positively to triglycerides $(p=0.014$, Fig. $2 \mathrm{a})$, CRP $(\mathrm{p}<0.001$, Fig. $2 \mathrm{~b})$, leukocyte blood count ( $<<0.001$, Fig. $2 c)$, and circulating TNF- $\alpha$ ( $p=0.004$, Fig. $2 d)$. No correlations between chemerin and the various parameters were found in control subjects.

\section{Chemerin levels are higher in patients with CAD compared to CAD-unaffected patients}

Chemerin levels did not differ between asymptomatic $(n=93)$ and symptomatic patients $(n=85$, Tab. 1$)$. We further examined patients with high grade stenosis $(\geq$ $90.0 \%$ luminal narrowing, $\mathrm{n}=85$ ). Symptomatic patients $(n=44)$ showed higher chemerin levels compared to asymptomatic patients $(n=41, p=0.014)$ with high grade stenosis.

50 out of 178 (50/178) patients were diagnosed for CAD. Patients with CAD had higher chemerin levels compared to CAD-unaffected patients $(n=128, p=0$. 002, Tab. 1). Construction of a ROC curve indicates that chemerin can determine whether patients had CAD or

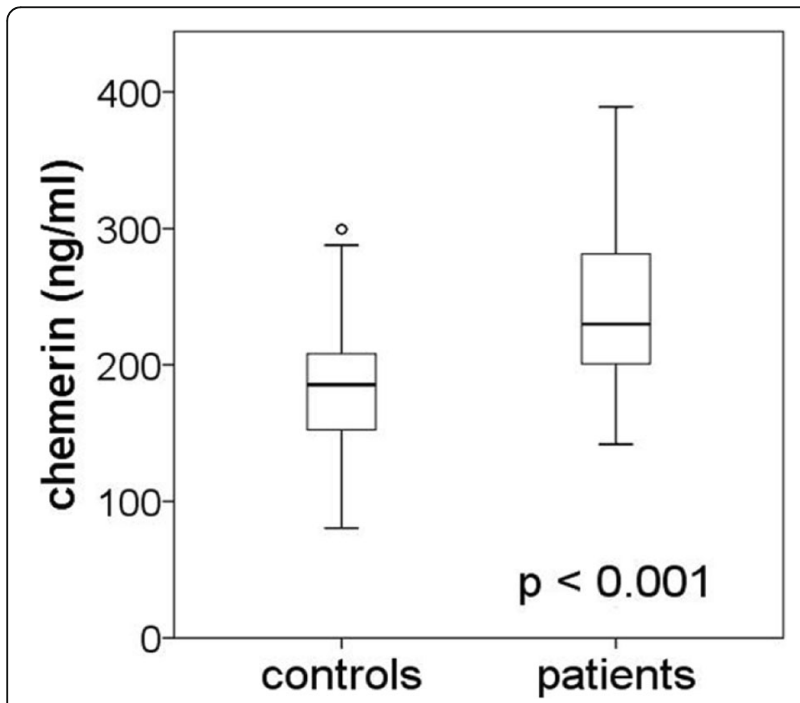

Fig. 1 Higher circulating chemerin in patients with advanced carotid stenosis. Chemerin levels differ between control subjects $(n=163)$ and patients ( $n=178$; median and interquartile range, whiskers 95\% percentile; t-test)
Table 2 Correlation of chemerin levels to anthropometric and metabolic characteristics of patients with carotid stenosis, significant differences are in bold

\begin{tabular}{lll}
\hline parameter & $r$ & $p$ \\
\hline age & 0.121 & 0.111 \\
BMl & 0.097 & 0.208 \\
HbA1c & 0.035 & 0.726 \\
fasting glucose & -0.024 & 0.780 \\
total cholesterol & -0.037 & 0.657 \\
LDL cholesterol & -0.064 & 0.440 \\
HDL cholesterol & -0.046 & 0.580 \\
triglycerides & 0.200 & $\mathbf{0 . 0 1 4}$ \\
leukocyte blood count & 0.309 & $<\mathbf{0 . 0 0 1}$ \\
CRP & 0.304 & $<\mathbf{0 . 0 0 1}$ \\
TNF-a & 0.360 & $\mathbf{0 . 0 0 4}$ \\
\hline
\end{tabular}

not (AUC 0.631 [0.535-0.724], $p=0.007$, Fig. 3). To further examine this hypothesis, we performed a logistic regression. Thereby we found that chemerin significantly increases the risk for CAD $(p=0.0013)$. Exactly one unit increase in chemerin increases the odds of having CAD by $0.2 \%$ (odds ratio $=1.002$ [1.001-1.003]).

\section{Discussion}

In the present study, we found higher circulating chemerin levels in patients with advanced carotid stenosis compared to age- and gender-matched controls.

Atherosclerosis is considered as chronic inflammation of the arteries [18]. Consistently, parameters of inflammation such as the CRP and leukocyte blood count were higher in our patients compared to controls. Both, CRP and the leukocyte blood count as well as circulating TNF- $\alpha$ correlated to chemerin in our patients confirming several studies in which chemerin and inflammatory parameters were strongly associated [19-23]. Chemerin directly affects the pathomechanism of atherosclerosis. Circulating chemerin increased after carotid balloon injury in mice, a model to induce atherosclerosis [11]. Chemerin siRNA knockdown inhibited the proliferation of vascular smooth muscle cells induced by plateletderived growth factor-BB and pro-inflammatory chemokines in vitro and prohibited carotid neointimal hyperplasia and pro-inflammatory chemokines in vivo after angioplasty [11]. Moreover, high levels of chemerin were associated with plaque instability, scored in histological sections [24]. This is likely due to the chemotactic effect of this adipokine on plaque macrophages, which changes the strength of the plaques. However, circulating chemerin was not associated with the cerebrovascular symptomatology of the patients [24], which is also the case in our study. Because the degree of carotid artery stenosis is an established risk factor for an ischemic 

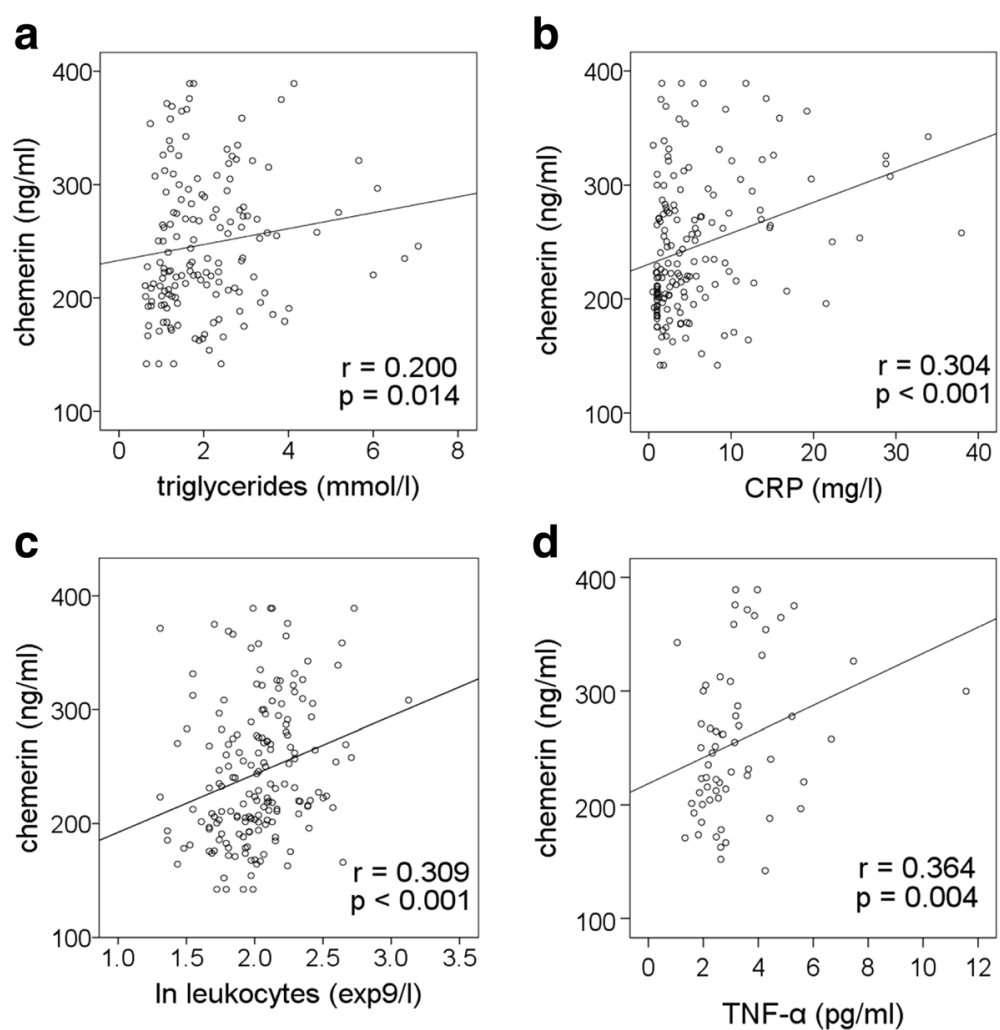

Fig. 2 Circulating chemerin correlates to triglycerides (a) and parameters of inflammation (b: C-reactive protein, CRP; c: peripheral leukocyte count; d: tumor-necrosis factor- $a$, TNF-a)

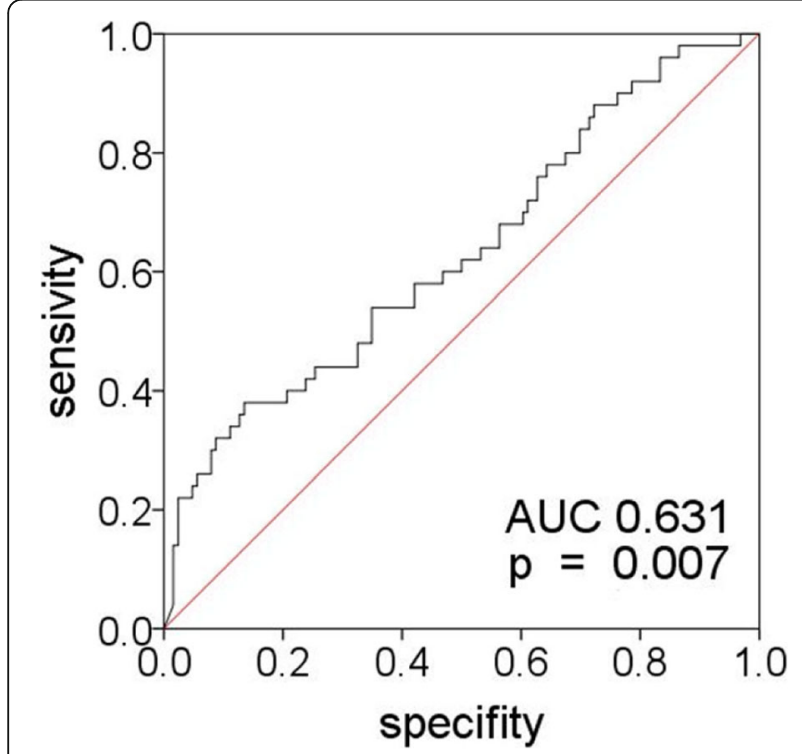

Fig. 3 AUC analysis of chemerin levels to predict coronary artery disease $(C A D)$ stroke [25], we re-evaluated patients with a high-grade stenosis concerning cerebrovascular symptomatology. Symptomatic patients had higher chemerin levels than asymptomatic patients with $\geq 90 \%$ luminal narrowing.

Our study confirmed the association of chemerin to CAD. Chemerin levels were elevated in metabolic syndrome patients with CAD [26] and positively related to the Gensini score, which evaluates the circulation in coronary arteries, in Chinese adults [27]. In a further study chemerin mRNA expression in epicardial adipose tissue was associated with coronary atherosclerosis [28] but in this study circulating chemerin and the Gensini score were not associated. Epicardial and periadventitial adipose tissue are not only innocent bystanders, but play a role in vascular pathophysiology through adipokine secretion. Indeed, periadventitial fat and foam cell chemerin immunopositivity correlated to the severity of atherosclerosis in human aortic and coronary artery samples [3]. In part, an autocrine positive feedback loop is established: both the adipokine and its receptor CMKLR1 are expressed in vascular smooth muscle and foam cells in atherosclerotic lesions of aortic and coronary vessels [3]. Interestingly, serum chemerin was found to be associated with the brachial ankle pulse wave velocity (baPWV), a parameter of arterial stiffness [22]. 
Adipose tissue is one of the major sources of chemerin [23]. Consistently, in several studies serum chemerin correlated to the BMI and waist circumference [21-23, 29]. In our study chemerin and the BMI were not associated. The BMI was even lower in patients compared to controls suggesting other cellular sources of chemerin such as the abdominal adipose tissue in patients with advanced carotid stenosis. Chemerin is widely distributed with particularly high levels in the liver [8] and, as discussed above, it is expressed in the affected vascular smooth muscle and foam cells of atherosclerotic lesions and fat enveloping arteries. Circulating, activated immune cells or thrombocytes, excreting chemerin during their activation [30], may be other cellular sources.

\section{Conclusions}

Circulating chemerin is elevated in patients with advanced carotid stenosis and CAD. It is related to parameters of systemic inflammation and in high grade stenosis to cerebrovascular symptomatology.

\section{Abbreviations}

AUC: Area under the ROC curve; BMI: Body mass index; CAD: Coronary artery disease; CEA: Carotid end arterectomy; CMKLR1: Chemokine-like receptor 1; CRP: C-reactive protein; HDL: High density lipoprotein; LDL: Low density lipoprotein; ROC: Receiver Operating Characteristic; T2DM: Type 2 diabetes mellitus; TNF: Tumor-necrosis factor

\section{Availability of data and materials}

The dataset used and analyzed during the current study is available from the corresponding author on reasonable request.

\section{Authors' contributions}

AK and CK performed assays. AK, MH and GA analyzed and interpreted the data. HS and NK contributed to data acquisition. GA wrote the manuscript. All authors have read and approved the final manuscript.

\section{Ethics approval and consent to participate}

This study was carried out in accordance with the recommendations of the Ethics Committee of the Medical Faculty of the Leipzig University (approvals no. 148-2005, 159-12-21,052,012, 017-12-23,012,012). All subjects gave written informed consent in accordance with the Declaration of Helsinki.

\section{Competing interests}

The authors declare that they have no competing interests.

\section{Publisher's Note}

Springer Nature remains neutral with regard to jurisdictional claims in published maps and institutional affiliations.

\begin{abstract}
Author details
${ }^{1}$ Research Laboratories; Clinic for Visceral, Transplantation, Thoracic and Vascular Surgery, Leipzig University, Liebigstr. 19, D-04103 Leipzig, Germany. ${ }^{2}$ Clinic for Visceral, Transplantation, Thoracic and Vascular Surgery, University Medical Centre Leipzig, Leipzig, Germany. ${ }^{3}$ Interdisciplinary Centre for Bioinformatics, Leipzig University, Leipzig, Germany. ${ }^{4}$ IFB Adiposity Disease, Junior Research Group 2, Leipzig University, Leipzig, Germany.
\end{abstract}

Received: 25 November 2017 Accepted: 4 April 2018 Published online: 13 April 2018

\section{References}

1. Dalen JE, Alpert JS, Goldberg RJ, Weinstein RS. The epidemic of the 20(th) century: coronary heart disease. Am J Med. 2014;127:807-12.
2. Thrift AG, Thayabaranathan T, Howard G, Howard VJ, Rothwell PM, Feigin VL, et al. Global stroke statistics. Int J Stroke. 2017;12:13-32.

3. Kostopoulos CG, Spiroglou SG, Varakis JN, Apostolakis E, Papadaki HH. Chemerin and CMKLR1 expression in human arteries and periadventitial fat: a possible role for local chemerin in atherosclerosis? BMC Cardiovasc Disord. 2014;14:56.

4. Mozaffarian D, Benjamin EJ, Go AS, Arnett DK, Blaha MJ, Cushman M, et al. Heart disease and stroke statistics-2016 update: a report from the american heart association. Circulation. 2016;133:e38-360.

5. Eckstein HH. Evidence-based management of carotid stenosis: recommendations from international guidelines. J Cardiovasc Surg. 2012;53:3-13.

6. Wittamer V, Franssen JD, Vulcano M, Mirjolet JF, Le PE, Migeotte I, et al. Specific recruitment of antigen-presenting cells by chemerin, a novel processed ligand from human inflammatory fluids. J Exp Med. 2003;198:977-85.

7. Kennedy AJ, Yang P, Read C, Kuc RE, Yang L, Taylor EJ, et al. Chemerin elicits potent constrictor actions via chemokine-like receptor 1 (CMKLR1), not G-protein-coupled receptor 1 (GPR1), in human and rat vasculature. J Am Heart Assoc. 2016;5:e004421.

8. Goralski KB, McCarthy TC, Hanniman EA, Zabel BA, Butcher EC, Parlee SD, et al. Chemerin, a novel adipokine that regulates adipogenesis and adipocyte metabolism. J Biol Chem. 2007;282:28175-88.

9. Watts SW, Dorrance AM, Penfold ME, Rourke JL, Sinal CJ, Seitz B, et al. Chemerin connects fat to arterial contraction. Arterioscler Thromb Vasc Biol. 2013;33:1320-8.

10. Lu B, Zhao M, Jiang W, Ma J, Yang C, Shao J, et al. Independent association of circulating level of chemerin with functional and early morphological vascular changes in newly diagnosed type 2 diabetic patients. Medicine. 2015;94:e1990. https://doi.org/10.1097/MD.0000000000001990.

11. Xiong W, Luo Y, Wu L, Liu F, Liu H, Li J, et al. Chemerin stimulates vascular smooth muscle cell proliferation and carotid neointimal hyperplasia by activating mitogen-activated protein kinase signaling. PLoS One. 2016;11: e0165305. https://doi.org/10.1371/journal.pone.0165305. eCollection 2016

12. Writing Group, Naylor AR, Ricco JB, de Borst GJ, Debus S, De HJ, et al. Editor's choice - management of atherosclerotic carotid and vertebral artery disease: 2017 clinical practice guidelines of the European Society for Vascular Surgery (ESVS). Eur J Vasc Endovasc Surg. 2018:55:3-81.

13. Brott TG, Halperin JL, Abbara S, Bacharach JM, Barr JD, Bush RL, et al. 2011 ASA/ACCF/AHA/AANN/AANS/ACR/ASNR/CNS/SAIP/SCAI/SIR/SNIS/SVM/SVS guideline on the management of patients with extracranial carotid and vertebral artery disease: executive summary: a report of the American College of Cardiology Foundation/American Heart Association task force on practice guidelines, and the American Stroke Association, American Association of Neuroscience Nurses, American Association of Neurological Surgeons, American College of Radiology, American Society of Neuroradiology, Congress of Neurological Surgeons, Society of Atherosclerosis Imaging and Prevention, Society for Cardiovascular Angiography and Interventions, Society of Interventional Radiology, society of Neurolnterventional surgery, Society for Vascular Medicine, and Society for Vascular Surgery. Developed in collaboration with the American Academy of Neurology and Society of Cardiovascular Computed Tomography. Catheter Cardiovasc Interv. 2013;81:E76-123.

14. Barnett HJM, Taylor DW, Haynes RB, Sackett DL, Peerless SJ, Ferguson GG, et al. Beneficial effect of carotid endarterectomy in symptomatic patients with high-grade carotid stenosis. N Engl J Med. 1991;325:445-53.

15. Kloting N, Fasshauer M, Dietrich A, Kovacs P, Schon MR, Kern M, et al. Insulin-sensitive obesity. Am J Physiol Endocrinol Metab. 2010;299:E506-15.

16. Aust G, Richter O, Rohm S, Kerner C, Hauss J, Klöting N, et al. Vaspin serum concentrations in patients with carotid stenosis. Atherosclerosis. 2009;204:262-6.

17. Aust G, Uptaite-Patapoviene M, Scholz M, Richter O, Rohm S, Bluher M. Circulating Nampt and RBP4 levels in patients with carotid stenosis undergoing carotid endarterectomy (CEA). Clin Chim Acta. 2011;412:1195-200.

18. Hansson GK. Inflammation, atherosclerosis, and coronary artery disease. N Engl J Med. 2005;352:1685-95.

19. Chakaroun R, Raschpichler M, Kloting N, Oberbach A, Flehmig G, Kern M, et al. Effects of weight loss and exercise on chemerin serum concentrations and adipose tissue expression in human obesity. Metabolism. 2012;61:706-14.

20. Weigert J, Neumeier M, Wanninger J, Filarsky M, Bauer S, Wiest R, et al. Systemic chemerin is related to inflammation rather than obesity in type 2 diabetes. Clin Endocrinol. 2010;72:342-8.

21. Lehrke M, Becker A, Greif M, Stark R, Laubender RP, Von ZF, et al. Chemerin is associated with markers of inflammation and components of the metabolic syndrome but does not predict coronary atherosclerosis. Eur J Endocrinol. 2009;161:339-44. 
22. Yoo HJ, Choi HY, Yang SJ, Kim HY, Seo JA, Kim SG, et al. Circulating chemerin level is independently correlated with arterial stiffness. J Atheroscler Thromb. 2012;19:59-66.

23. Bozaoglu K, Bolton K, McMillan J, Zimmet P, Jowett J, Collier G, et al. Chemerin is a novel adipokine associated with obesity and metabolic syndrome. Endocrinology. 2007:148:4687-94.

24. Gasbarrino K, Mantzoros C, Gorgui J, Veinot JP, Lai C, Daskalopoulou SS. Circulating chemerin is associated with carotid plaque instability, whereas resistin is related to Ccrebrovascular symptomatology. Arterioscler Thromb Vasc Biol. 2016;36:1670-8.

25. Fairhead JF, Rothwell PM. The need for urgency in identification and treatment of symptomatic carotid stenosis is already established. Cerebrovasc Dis. 2005;19:355-8.

26. Dong B, Ji W, Zhang Y. Elevated serum chemerin levels are associated with the presence of coronary artery disease in patients with metabolic syndrome. Intern Med. 2011;50:1093-7.

27. Yan Q, Zhang Y, Hong J, Gu W, Dai M, Shi J, et al. The association of serum chemerin level with risk of coronary artery disease in Chinese adults. Endocrine. 2012;41:281-8.

28. Gao X, Mi S, Zhang F, Gong F, Lai Y, Gao F, et al. Association of chemerin mRNA expression in human epicardial adipose tissue with coronary atherosclerosis. Cardiovasc Diabetol. 2011:10:87.

29. Kunimoto H, Kazama K, Takai M, Oda M, Okada M, Yamawaki H. Chemerin promotes the proliferation and migration of vascular smooth muscle and increases mouse blood pressure. Am J Physiol Heart Circ Physiol. 2015;309:H1017-28.

30. Du XY, Zabel BA, Myles T, Allen SJ, Handel TM, Lee PP, et al. Regulation of chemerin bioactivity by plasma carboxypeptidase $\mathrm{N}$, carboxypeptidase B (activated thrombin-activable fibrinolysis inhibitor), and platelets. J Biol Chem. 2009;284:751-8.

Ready to submit your research? Choose BMC and benefit from:

- fast, convenient online submission

- thorough peer review by experienced researchers in your field

- rapid publication on acceptance

- support for research data, including large and complex data types

- gold Open Access which fosters wider collaboration and increased citations

- maximum visibility for your research: over $100 \mathrm{M}$ website views per year

At BMC, research is always in progress.

Learn more biomedcentral.com/submissions 